\title{
Lagrangian coherent structures in the flow field of a fluidic oscillator
}

\author{
Moritz Sieber, ${ }^{*}$ Florian Ostermann, Rene Woszidlo, Kilian Oberleithner, and C. Oliver Paschereit \\ Institut für Strömungsmechanik und Technische Akustik, HFI, Technische Universität Berlin, \\ 10623 Berlin, Germany
}

(Received 10 August 2016; published 12 September 2016)

\begin{abstract}
This paper is associated with a video winner of a 2015 APS/DFD Gallery of Fluid Motion Award. The original video is available from the Gallery of Fluid Motion, http://dx.doi.org/10.1103/APS.DFD.2015.GFM.V0015
\end{abstract}

DOI: 10.1103/PhysRevFluids.1.050509

Fluidic oscillators are flow devices that generate a spatially oscillating jet without any moving parts. They are also known as sweeping jet actuators because the generated jet sweeps from side to side in a windshield wiper manner (Fig. 1). The absence of moving parts renders these devices very reliable and robust for flow control applications, such as separation control, noise control, combustion control, and drag reduction [1]. In order to develop design rules and scaling laws for these highly effective actuators, extensive fundamental studies have been conducted within our collaborative groups. Woszidlo et al. [2] describe the internal and external flow field of a fluidic oscillator emitting its jet into a quiescent environment. Von Gosen et al. [3] investigate compressibility effects inside and outside an oscillator. Ostermann et al. [4] compare two commonly used oscillator designs by means of high-speed particle image velocimetry (PIV). The data from the latter study forms the basis for the video.

This short article provides some details on the underlying experiments and methods. The video shows Lagrangian coherent structures (LCSs), which are identified and visualized by the finite-time Lyapunov exponent (FTLE) [5]. These LCSs describe attracting or repelling material surfaces of the flow, which are particularly suited for intuitive visualizations of flow structures.

The data are acquired by PIV on a large-scale fluidic oscillator as described by Ostermann et al. [4]. In order to increase the spatial resolution, the flow field is combined from several individually recorded segments. The flow field is phase-averaged with respect to a pressure reference signal [6]. Another recently developed flow decomposition method shows precise separation solely from PIV data [7]. Phase averaging eliminates stochastic noise and turbulence and yields a representative oscillation period. The phase-averaged flow data are used to calculate the separation of convected particles, which provides the FTLE measure. In particular, the backward-time FTLE is computed over one period.

Figure 1(a) shows a photograph of a smoke visualization. This simple experimental technique illustrates the sweeping motion of the emanating jet, but it does not reveal the flow dynamics inside the fluidic device. Figure 1(b) illustrates the PIV results showing contours of the phase-averaged velocity magnitude-an Eulerian representation of the flow field dynamics. Flow structures in the diverging center channel and the bypass channels are distinguishable, but they appear blurry and unspecific. Switching to the Lagrangian viewpoint, the FTLE shown in Fig. 1(c) visualizes the coherent structures at a much higher level of detail. We see two head vortices that are created when the jet is at its maximum deflection and convected downstream. Inside the oscillator, the main flow as well as a large separation bubble is revealed. The chronology of flow states in the video illustrate

\footnotetext{
*moritz.sieber@fd.tu-berlin.de
}

Published by the American Physical Society under the terms of the Creative Commons Attribution 3.0 License. Further distribution of this work must maintain attribution to the author(s) and the published article's title, journal citation, and DOI. 
MORITZ SIEBER et al.

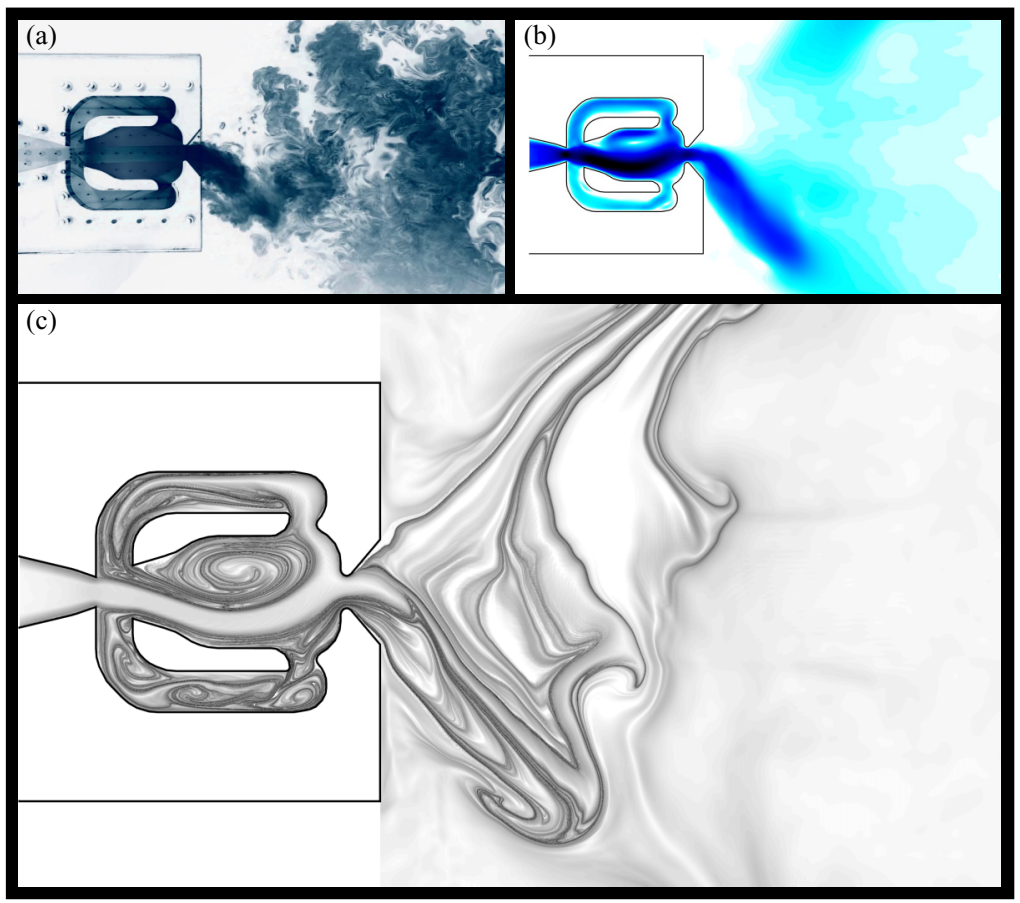

FIG. 1. Investigating the flow field of a fluidic oscillator. (a) Smoke visualization, (b) Eulerian representation of PIV data, and (c) finite-time-Lyapunov exponent. The video associated with these images is available at http://dx.doi.org/10.1103/APS.DFD.2015.GFM.V0015

the flow feedback occurring inside the actuator and the sweeping motion of the jet. The close-ups shown in the video provide an intuitive understanding of how the flapping motion is generated.

This example shows that the FTLE is a valuable tool for identifying coherent structures and illustrating complex flow dynamics. The FTLE reveals more information and is closer to our visual interpretation of flow dynamics than the Eulerian representation of flow quantities as it considers the temporal history of the flow field. It provides an intuitive understanding due to the affinity to transport related structures known from classical dye or smoke flow visualizations.

The authors kindly acknowledge the funding from the German Research Foundation (DFG Project PA 920/30-1).

[1] J. W. Gregory and M. N. Tomac, Proceedings of the 43rd AIAA Fluid Dynamics Conference (AIAA, Reston, 2013).

[2] R. Woszidlo, F. Ostermann, C. N. Nayeri, and C. O. Paschereit, The time-resolved natural flow field of a fluidic oscillator, Exp. Fluids 56, 125 (2015).

[3] F. Von Gosen, F. Ostermann, R. Woszidlo, C. N. Nayeri, and C. O. Paschereit, Proceedings of the 53rd AIAA Aerospace Sciences Meeting (AIAA, Reston, 2015).

[4] F. Ostermann, R. Woszidlo, C. N. Nayeri, and C. O. Paschereit, Proceedings of the 53rd AIAA Aerospace Sciences Meeting (Ref. [3]).

[5] G. Haller, Lagrangian coherent structures, Annu. Rev. Fluid Mech. 47, 137 (2015).

[6] F. Ostermann, R. Woszidlo, C. N. Nayeri, and C. O. Paschereit, Phase-averaging methods for the natural flowfield of a fluidic oscillator, AIAA J. 53, 2359 (2015).

[7] M. Sieber, C. O. Paschereit, and K. Oberleithner, Spectral proper orthogonal decomposition, J. Fluid Mech. 792, 798 (2016). 